\title{
Clinical outcomes of polymeric sealant use in pulmonary resection: a systematic review and meta-analysis of randomized controlled trials
}

\author{
Anna L. McGuire ${ }^{1,2}$, John Yee Y,2 $^{1,2}$ \\ ${ }^{1}$ Division of Thoracic Surgery, University of British Columbia, Vancouver, British Columbia, Canada; ${ }^{2}$ Vancouver Coastal Health Research Institute, \\ Vancouver General Hospital, Vancouver, Canada \\ Contributions: (I) Conception and design: AL McGuire; (II) Administrative support: None; (III) Provision of study materials or patients: None; (IV) \\ Collection and assembly of data: All authors; (V) Data analysis and interpretation: All authors; (VI) Manuscript writing: All authors; (VII) Final \\ approval of manuscript: All authors. \\ Correspondence to: Anna L. McGuire, MD, MSc, FRCSC. Division of Thoracic Surgery, University of British Columbia, Vancouver Coastal Health, \\ 7113-2775 Laurel Street, Vancouver, BC V5Z 1M9, Canada. Email: anna.mcguire@vch.ca.
}

Background: Prolonged alveolar air leak (PAL) is the most common adverse event following pulmonary resection. It carries morbidity for patients by increasing empyema risk, and for hospital administration with the cost of prolonged length of hospital stay (LOS). Intra-operative sealant technology is available to surgeons, and may decrease PAL. Our aim was to conduct a systematic review and meta-analysis of randomized controlled trials (RCTs) on the effect of intraoperative polymeric sealant use on PAL, empyema, and LOS.

Methods: Standard PRISMA (Preferred Reporting Items for Systematic reviews and Meta-analysis and methods) protocol was adhered to. For qualitative review the search strategy yielded 21 RCTs reporting polymeric sealant use in lung resection, 19 of which were included in meta-analyses. The control arm in the two excluded RCTs was not "standard care." Random-effects meta-analyses were conducted. Inter-trial heterogeneity was assessed with the I2 statistic. Publication bias was assessed with a funnel plot and Egger statistic for small study effects.

Results: Pooled analysis was derived from 2,537 randomized participants. They were allocated to the intervention arm of lung resection with intra-operative application of a polymeric sealant $(\mathrm{n}=1,292)$, or the control arm of standard care involving pulmonary resection with pneumostasis by sutures and/or stapler $(\mathrm{n}=1,245)$. Participants came from 10 different countries, with mean (SD) age of 62.5 (4.2) years, and 31.6\% (95\% CI: 30.0-33.5) female. Pooled estimates revealed polymeric sealant decreased odds of PAL (OR 0.55, 95\% CI: 0.35-0.87), and decreased LOS by one day (mean difference -0.96 (95\% CI: -1.74 to -0.18 ), without increasing odds of pleural sepsis (OR 1.134, 95\% CI: 0.343-3.748). There was evidence of publication bias in the LOS meta-analysis.

Conclusions: Pooled analysis revealed decreased odds of PAL, and decreased LOS by one day with intraoperative use of polymeric sealants. There was no associated increase in odds of adverse events, including empyema.

Keywords: Lung cancer, lobectomy, air leak, fibrin, empyema

Submitted May 02, 2018. Accepted for publication Oct 15, 2018.

doi: $10.21037 /$ jtd.2018.10.48

View this article at: http://dx.doi.org/10.21037/jtd.2018.10.48 


\section{Introduction}

Prolonged alveolar air leak (PAL) is the most common complication following pulmonary resection, both anatomic (lobectomy, segmentectomy) and non-anatomic (wedge resection). High volume thoracic surgical institutions report a PAL incidence of at least $15 \%$ annually (1-3). PAL has known significant associated morbidity for lung resection patients, in the form of pain from prolonged chest tube drainage, an increased empyema risk (3-5). Even if a thoracic surgical service has a program whereby PAL patients can be safely discharged with a Pneumostat ${ }^{\circledR}$ or Heimlich valve enabling the air leak to be followed and chest drain removed as an outpatient, PAL still represents an additional hospital expense $(6,7)$. The metric of additional cost for hospital administration is captured not only with increased length of index stay (LOS) related to the lung resection, but also for additional outpatient followup visits for chest tube trouble-shooting, and potential hospital readmission for management of pleural sepsis (5).

Because PAL is a common and costly adverse event, there has been much prospective investigation on risk factor identification and thus prevention, both at the patientlevel and surgical technique level. Patient-level risk factors have been identified in previous studies and well described. Much of our existing knowledge on air leak risk factors and management comes from the lung volume reduction (LVRS) literature, including the National Emphysema Treatment Trial (NETT) (8-10). Factors shown from NETT and other study cohorts to place a patient at higher than baseline risk for PAL include: emphysema, immune suppression, corticosteroid use, previous thoracotomy, pleural adhesions, and incomplete inter-lobar fissure (1,6,10-14).

Thoracic surgeons have also endeavored decrease PAL with intra-operative technical modifications. Blood patch, pleural tenting, pneumoperitoneum and phrenic nerve paralysis have reported mixed efficiency (15-18). When approaching an incomplete lobar fissure, the "fissureless" approach employing surgical stapling has shown to result in greater pneumostasis compared to precision dissection with electrocautery $(16,19)$. Additionally, buttress strips made of gortex or bovine pericardium have been used with staplers as a mechanical sealant in an effort to enhance pneumostasis in the setting of LVRS as well as incomplete fissure at the time of lobectomy $(20,21)$.

Application of polymerizing sealant products at the time of surgery (via liquid or soft collagen fleece patch) over staple lines and areas of cautery dissection are another attractive method for thoracic surgeons to decrease air leak and PAL. These products initially gained popularity for hemostatic indications in cardiac and vascular surgery, subsequently finding pneumostatic utility in thoracic surgery. In general, the polymerizing sealants can be classified by their constituent derivation as synthetic hydrogels or biologic (fibrin or albumin based). Fibrin based sealants are further sub-classified by source of thrombin and fibrinogen to form the fibrin polymer: human derived (homologous and autologous options exist), versus bovine or equine derived. Several small-scale prospective randomized controlled trials (RCTs) have been conducted examining the role of these polymerizing sealants in clinical outcomes of: PAL incidence, duration of air leak, duration of chest drains, and occurrence post-operative adverse events such as empyema (2).

A Cochrane Systematic Review of published and nonpublished RCTs of sealants to prevent air leak following lung surgery was conducted in 2010 (2). This analysis grouped together polymeric and mechanical sealant methods, which have quite different pneumostatic mechanisms. The review found that further large RCTs were required to clearly determine effects of all surgical sealants on the outcomes outlined above, especially LOS. The individual patient data RCT review found surgical sealants decrease air leak volume, however some possibly increase risk of pleural space infection. Although powered to detect differences in PAL and time to chest drain removal, individual studies primary outcomes have not been LOS, and thus have been underpowered to detect statistically meaningful differences in these secondary outcomes (2).

A systematic review and meta-analysis of surgical sealants effect on PAL in the setting of LVRS was also conducted in 2010 by Malapert and colleagues (22). Similar to the Cochrane Systematic review, this meta-analysis grouped together mechanical and polymerizing surgical sealant methods. The pooled analysis did find that use of surgical sealant reduced odds of PAL following pulmonary resection, without increased odds of pleural space infection. To explore heterogeneity between trials they did conduct a stratified analysis by sealant type. LOS and other continuous outcomes did not undergo a pooled analysis, and some smaller scale RCTs were not included $(23,24)$.

Additionally, in the years since publication of these reviews in 2010, there have been more RCTs conducted examining the effects of polymerizing surgical sealants on air leak after lung resection in the era of video-assisted thoracic surgical (VATS) technology. 
The aim of this systematic review and meta-analysis is thus to provide an update on the evidence of effects of polymeric surgical sealant use from published RCTs on the primary outcomes of PAL and LOS. Secondary outcomes of interest include adverse events such as empyema, duration of air leak, and duration of indwelling chest drains.

\section{Methods}

This systematic review and meta-analysis were guided by the standard PRISMA protocol (Preferred Reporting Items for Systematic reviews and Meta-analysis and methods) proposed by the Cochrane Collaboration $(25,26)$.

Using a pre-defined key-word search strategy, the Ovid electronics databases MEDLINE, and the Cochrane Central Register of Controlled Trials (CENTRAL) were queried from 1948 to 2018. The search was limited to human subjects and to English language full-text availability studies. To identify all articles possible for inclusion, a manual search of references in all original articles as well as review papers was conducted.

An electronic systematic search of the medical literature was performed in February 2018 to identify all published prospective RCTs in which standard surgical closure techniques (suturing/stapling) was conducted with polymeric sealant products in pulmonary resections, compared to the same intervention without use of polymerizing sealant. The polymerizing sealant could be delivered in the form of a liquid spray, or a soft patch. For this study we defined PAL as an air-leak persisting for $\geq 5$ post-operative days (27).

\section{Data collection and analysis}

Two reviewers independently selected trials for inclusion in the review, assessed methodology quality of each, and extracted pre-determined data with a standardized form.

Analysis was performed using Stata12 statistical software (version 12, College Station, TX). For continuous variables, the mean difference with standard deviation (SD) between intervention and control groups were obtained for each study with $95 \%$ confidence intervals (CI) and pooled via random-effects meta-analysis. For categorical variables, odds of events and non-events were obtained to generate odds ratios with $95 \%$ CIs for each study and combined with a random-effects model. A $\mathrm{P}<0.05$ was considered to indicate strong evidence of association between variables. Extracted data was pooled with weighted averages, where the weight of each study was its sample size.
Formal assessment of between study heterogeneity was conducted with the $\mathrm{I}^{2}$ statistic; the proportion of total variation across studies due to between study heterogeneity. With a range between $0 \%$ and $100 \%$, the $\mathrm{I}^{2}$ statistic was taken to indicate moderate between study heterogeneity between studies at a value of $50 \%$, and high heterogeneity at a value of $75 \%$ (28).

Publication bias was formally assessed with a funnel plot and the Egger test, where $\mathrm{P}<0.05$ was taken to indicate strong evidence supporting presence of publication bias (29). Begg's statistic was additionally employed to assess for small study effects.

\section{Results}

\section{Study selection}

The systematic search yielded 54 articles (Figure 1). Following removal of duplicates, 44 articles were selected for full text review of which 14 studies were identified from manual reference search. Abstract review was conducted on the 44 records. Of these, 23 were excluded because they did not report a polymerizing sealant, employed a nonpulmonary use of the sealant, or were not prospective RCTs (review articles or editorial commentaries). This left 21 studies for full text review. All of these were included in the qualitative synthesis. Two RCTs were excluded from the meta-analysis, for a total of 19 considered in the pooled analysis, because the control group was not "standard care" of air leak with surgical pneumostasis via sutures and/or staplers.

\section{Study description}

Main included trial characteristics are outlined in Table 1. This systematic review and meta-analysis involved a total of 2,537 participants randomized to the intervention group of pulmonary resections with intra-operative application of a polymeric sealant in addition to standard care $(\mathrm{n}=1,292)$, or the control group of standard care (pulmonary resection with pneumostasis by sutures and/or stapler, $n=1,245$ ).

The mean (SD) age of participants was 62.5 (4.2) years [sealant 62.2 (4.8), control 63.0 (3.9)]. A total of 31.6\% (95\% CI: $30.0-33.5 \%$ ) were female (sealant $31.6 \%$, $95 \%$ CI: 29.1-34.2\%; control 31.6\%, 95\% CI: 29.1-34.4\%). Study participants came from 10 different countries.

Synthetic polymeric sealants were used in 6 trials (30-35), 13 studies used fibrin-based sealants $(23,24,36-46)$, and 


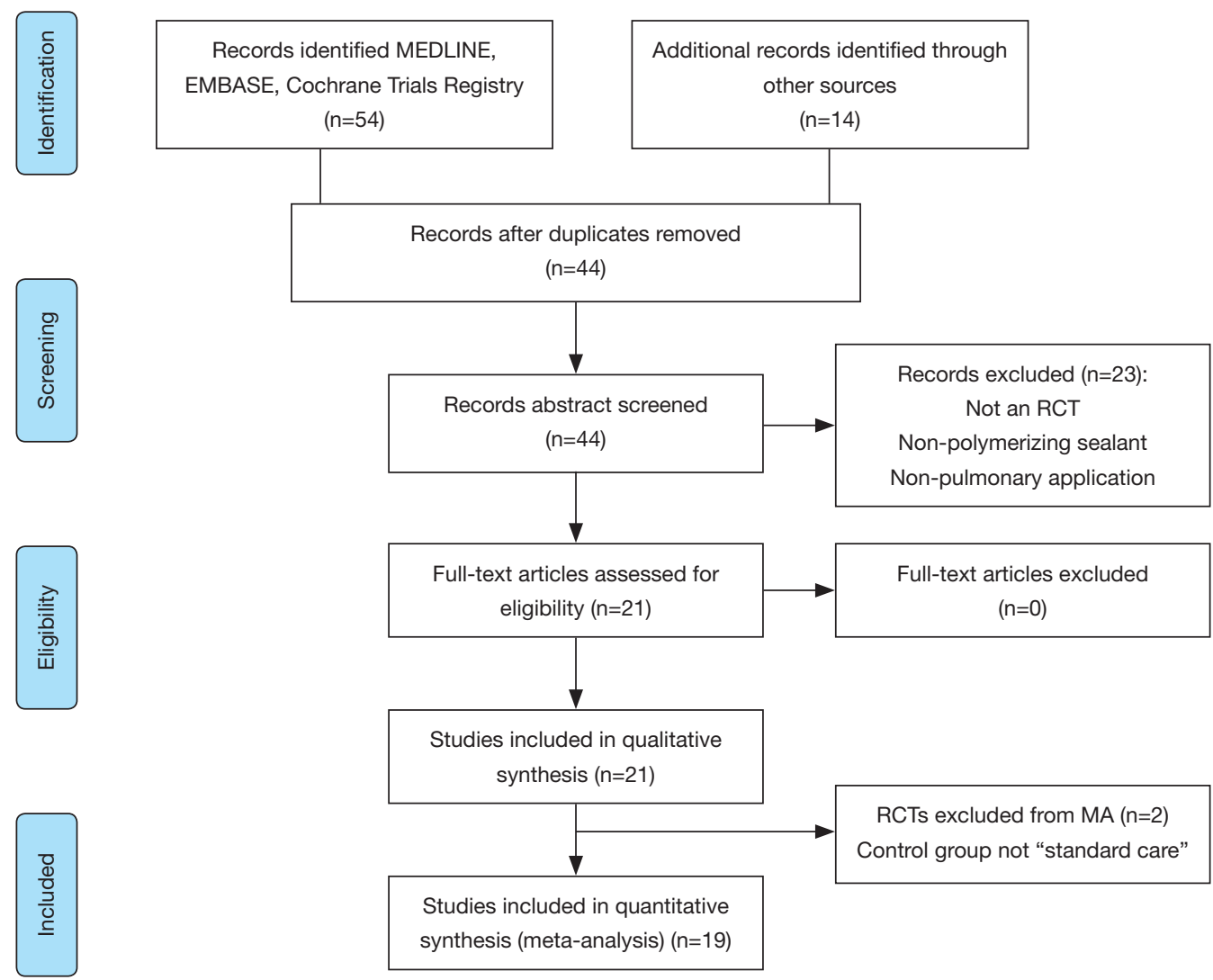

Figure 1 PRISMA flow diagram. RCT, randomized controlled trial; MA, meta-analysis.

2 studies used other biologic (albumin based) sealants $(47,48)$.

\section{Trial design quality}

Figure 2 outlines important quality metrics to consider in these trials. The vast majority of trials described adequate concealment of allocation methods. The majority were also open-label without investigator blinding to allocation group. This is in large part due to the nature of the surgical intervention under study, making observer blinding exceedingly difficult to achieve. It is unclear in the majority of included trials as to whether the study participant was aware of the treatment arm to which they were allocated. No study reported whether blinded statistical analysis was conducted. Most trials used analog chest drainage system to quantify presence and severity of air leak. Compared to digital chest drainage systems, more inter-observer variability in air leak assessment is an issue with analog systems (49). Most studies did not require a standardized post-operative chest tube management algorithm to be adhered to in their trial protocol, which may affect precision of individual estimates.

\section{$P A L$}

Twelve RCTs contributed to the meta-analysis of intraoperative polymerizing sealant effect on odds of PAL (Figure 3). The pooled odds ratio was 0.549 (95\% CI: $0.35-0.87)$. Between-trial heterogeneity was low with $\mathrm{I}^{2}$ of $27.5 \%, \mathrm{P}=0.175$.

Publication bias assessment with the funnel plot revealed a lack of large studies with high precision (Figure 4) in the PAL meta-analysis. The Begg's statistic for small study effect was $\mathrm{P}=0.01$, providing good evidence consistent with funnel plot assessment, that the meta-analysis comprised small studies overall with lower baseline precision estimates.

\section{LOS}

Nine RCTs contributed to the meta-analysis of intraoperative polymerizing sealant effect on mean difference in LOS (Figure 5). The pooled mean difference for LOS 


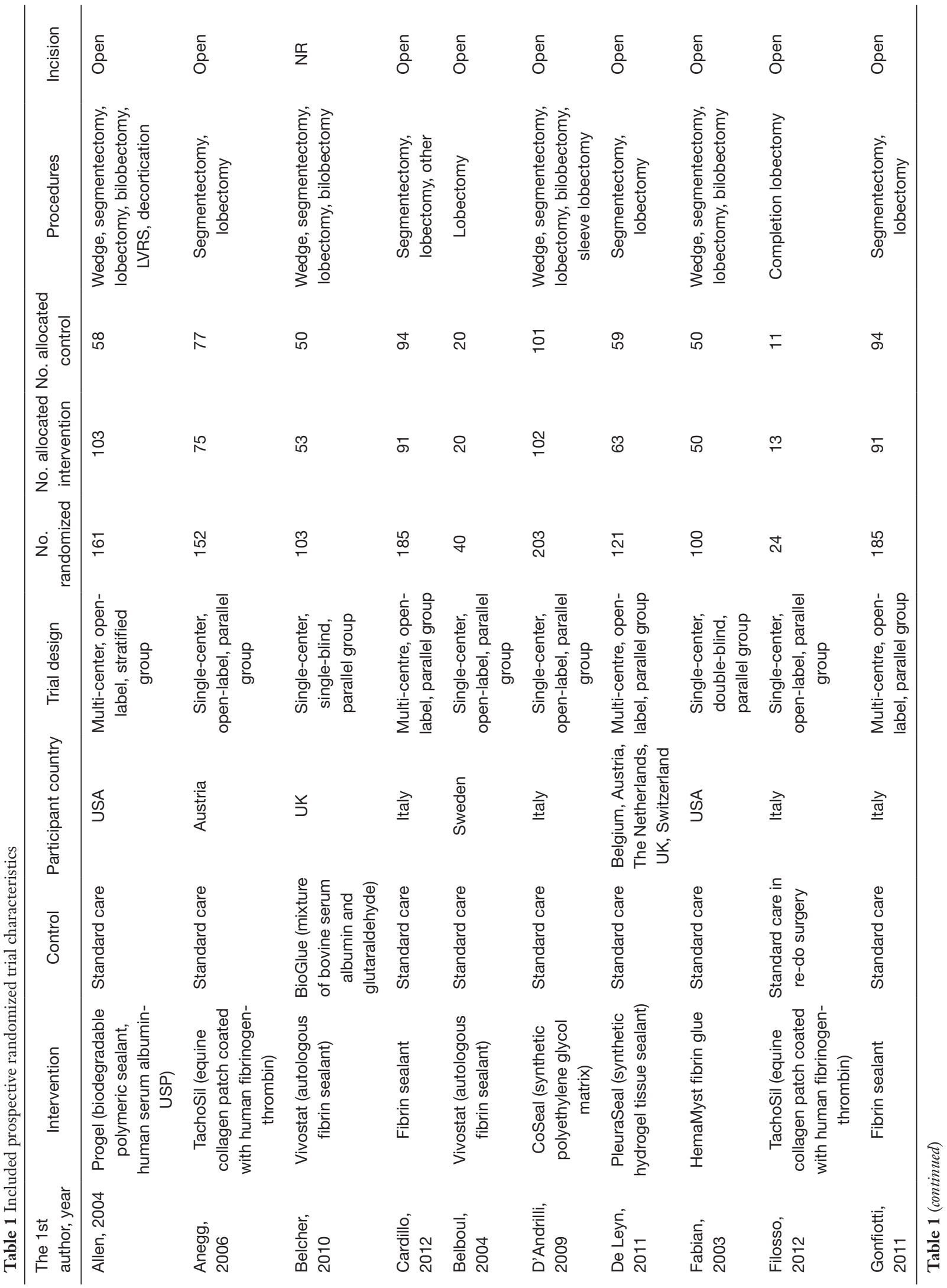




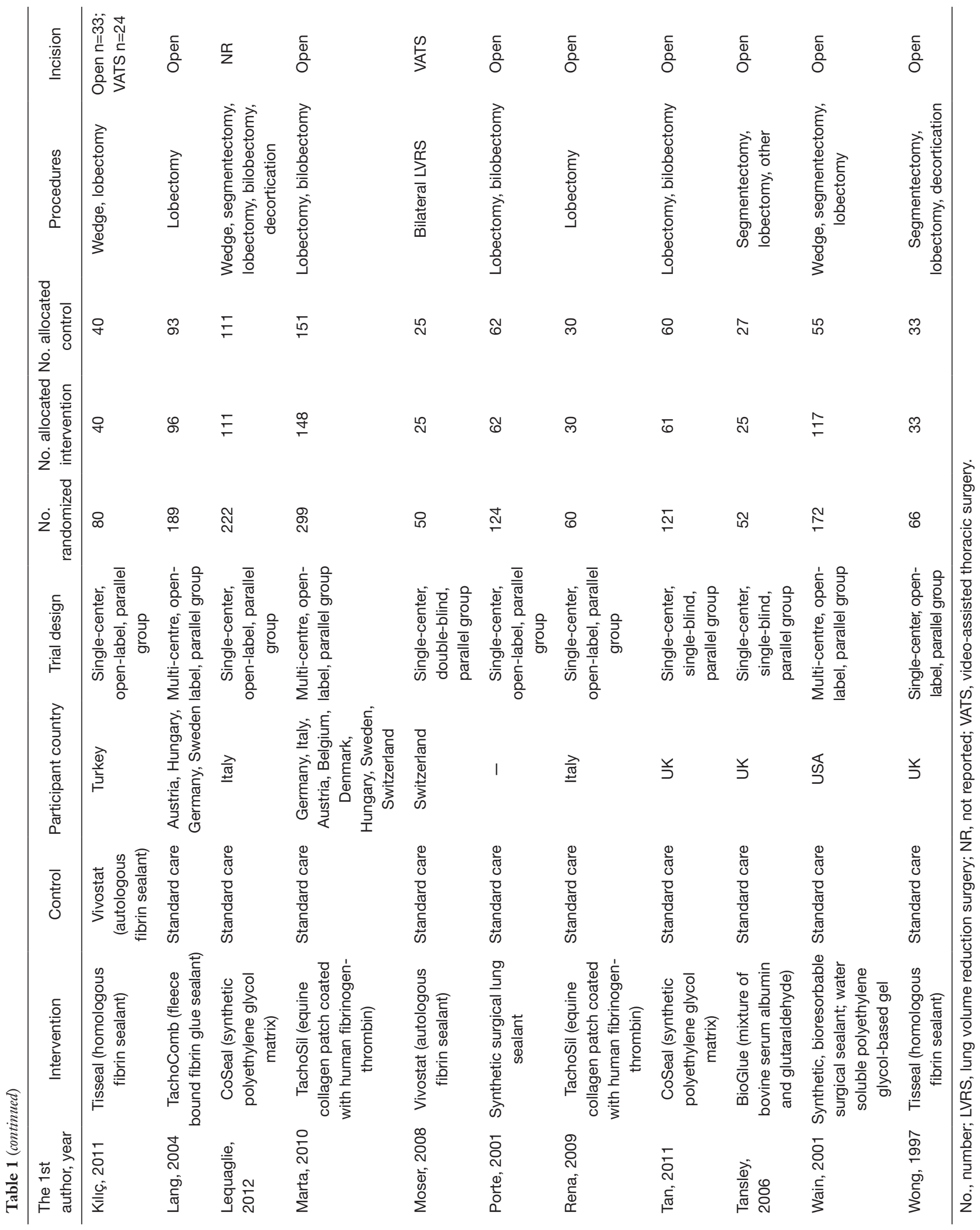




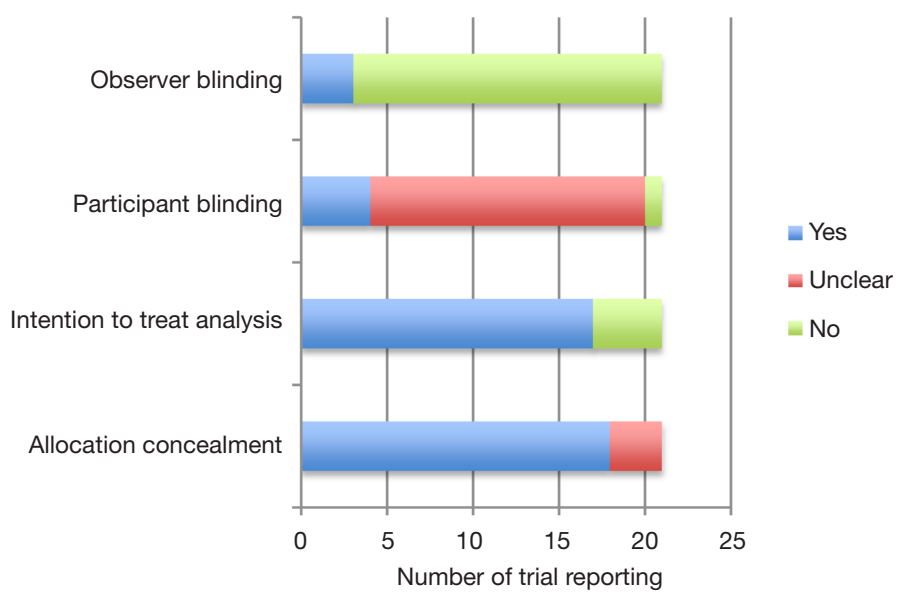

Figure 2 Randomized trial quality metrics of 21 included studies.

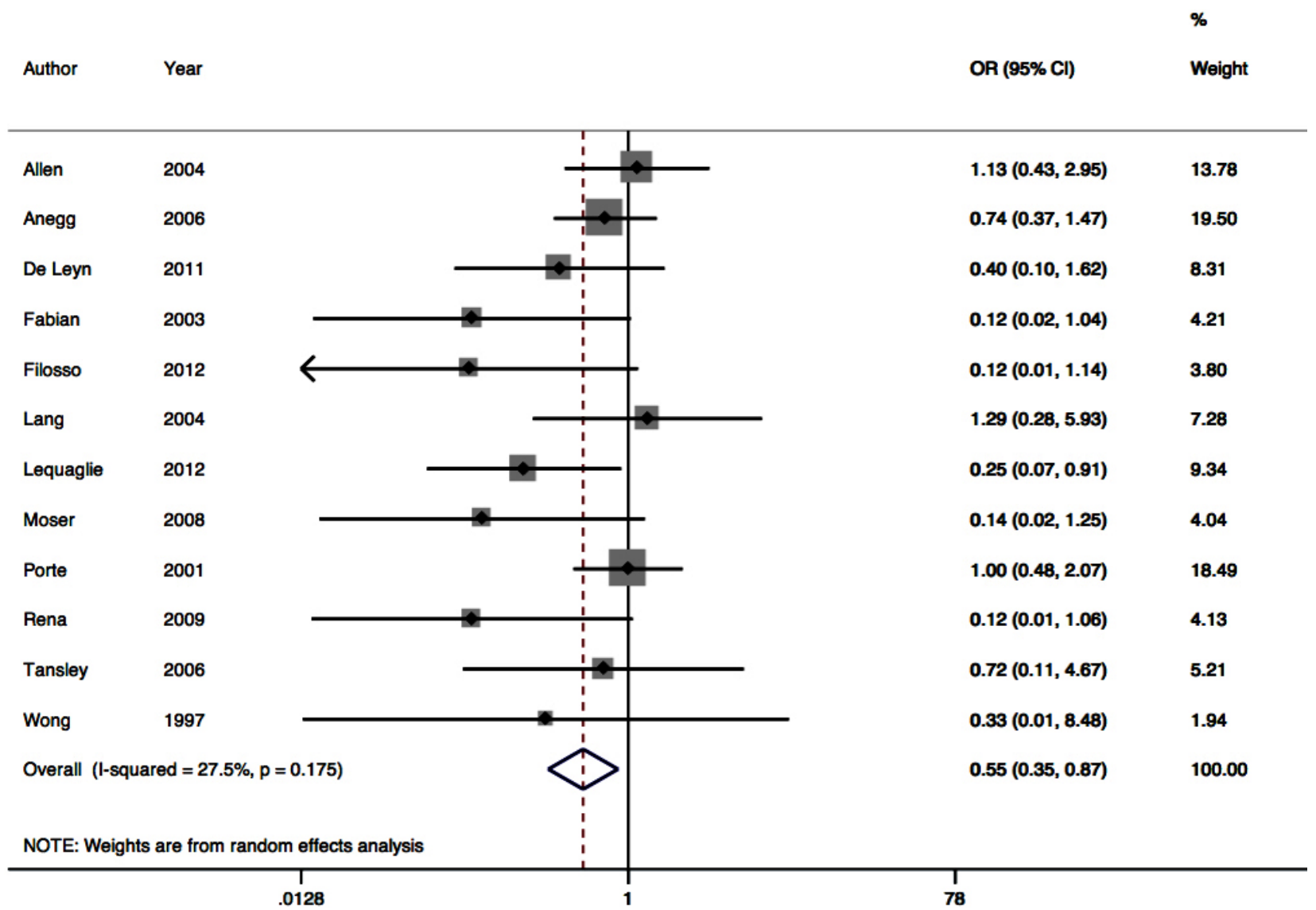

Figure 3 Prolonged alveolar air leak forest plot.

was one day in hospital, or -0.96 (95\% CI: -1.74 to -0.18$)$. Between-trial heterogeneity was very high with $\mathrm{I}^{2}$ of $97.4 \%$ $\mathrm{P}=0.001$.

Publication bias assessment with the funnel plot revealed most studies contributing to the LOS estimates were larger studies, possibly with higher precision (Figure 6). The Begg's statistic for small study effect revealed $\mathrm{P}=0.166$ which was consistent with the funnel plot. Additionally, 
the funnel plot revealed several outliers, making it appear asymmetric despite higher precision of individual studies. This is consistent with the finding of high level of inter-trial heterogeneity on $\mathrm{I}^{2}$ statistic for LOS.

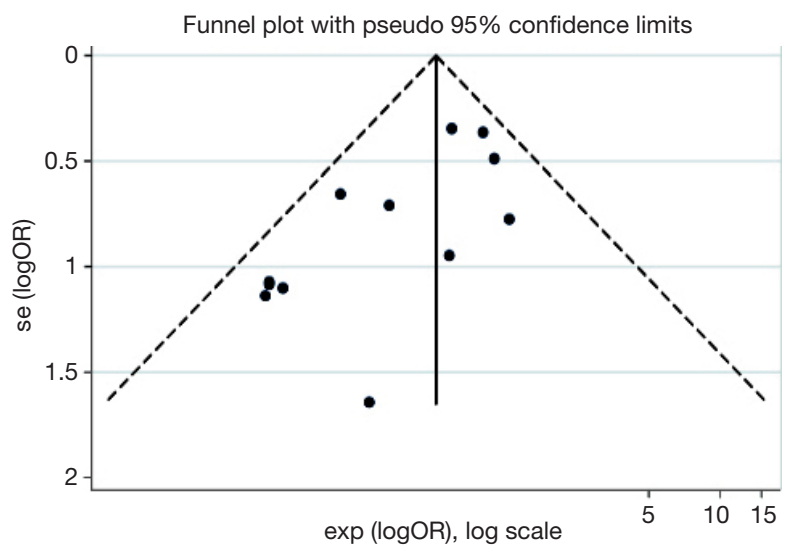

Figure 4 Funnel plot for publication bias and small study effects in trials reporting prolonged air leak. OR, odds ratio.

\section{Duration of air leak and indwelling chest drains}

Pooled estimates of 9 trials reporting mean duration of air leak, and 7 trials on duration of chest tube drainage, both revealed strong evidence of a shorter duration of one day with sealant $(\mathrm{P}<0.001)$ (Table 2$)$. There was however in both pooled analyses very high levels of inter-RCT heterogeneity, with $\mathrm{I}^{2}$ of $95.3 \%$ and $89.9 \%$ respectively.

\section{Adverse events}

Across all pooled analyses for reported adverse events in the trials, heterogeneity was very low, with $\mathrm{I}^{2}$ ranging from $0 \%$ to $27.5 \%$ (Table 3 ). The most common adverse events after pulmonary resection in these trials included: prolonged air leak, atrial arrhythmia, pneumothorax, pneumonia, hemothorax, atelectasis, and empyema. Metaanalysis of 7 reporting RCTs revealed no increased odds of empyema with use of polymerizing sealants OR 1.134 (95\% CI: 0.343-3.748; $\mathrm{P}=0.837)$. Similarly, odds of death were

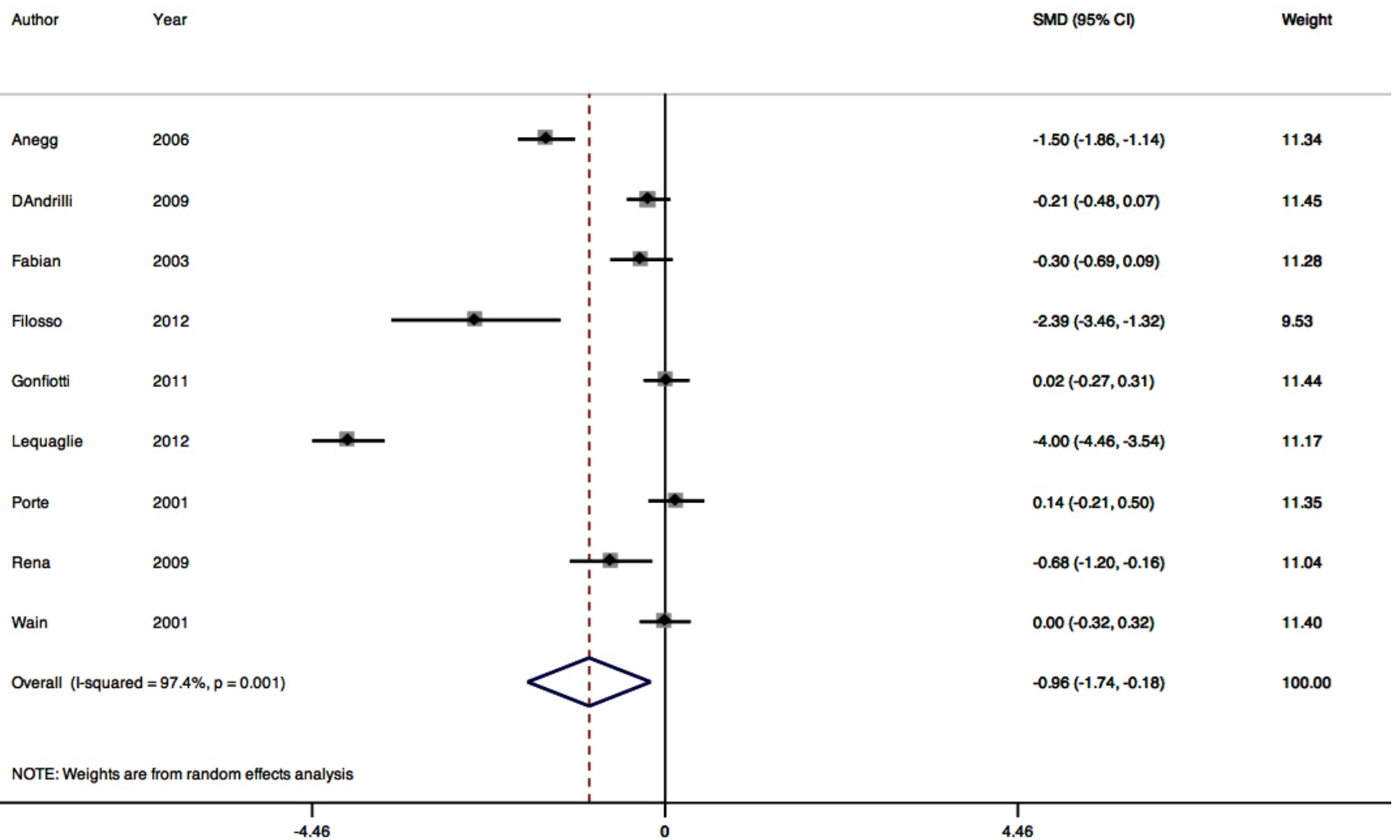

Figure 5 Length of stay in hospital forest plot. 
not increased with use of sealants, in fact it was somewhat protective on this pooled analysis, with OR 0.447 (95\% CI: $0.228-0.877 ; \mathrm{P}=0.019)$. The pooled odds ratio and $\mathrm{I}^{2}$ statistic for the other most common adverse event reported are summarized in Table 3. None revealed increased odds of an adverse event with use of surgical sealant compared to controls.

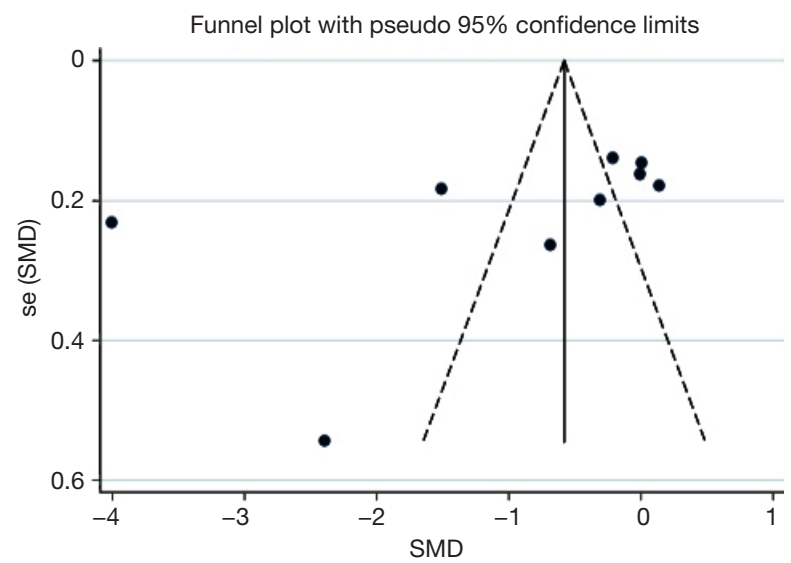

Figure 6 Length of stay in hospital funnel plot for publication bias and small study effects.

\section{Discussion}

This systematic review and meta-analysis of RCTs is unique in that it focuses to the role of polymeric surgical sealants. There are findings in our study that are novel, in addition to those that support existing data. There is strong evidence in the pooled analysis that odds of PAL is significantly reduced when a polymeric surgical sealant is applied intraoperatively (OR 0.5, 95\% CI: 0.3-0.9). There was additionally minimal between trial heterogeneity demonstrated $\mathrm{I}^{2}=27.5, \mathrm{P}=0.01$. This clinically relevant finding regarding PAL is consistent with that of Malapert and colleagues (22).

There is also moderate evidence, despite inter-trial trial heterogeneity, that the duration of an air leak, duration of the chest tube, and LOS are all reduced by an average of one day with polymeric sealant use (Table 2). Possible reasons for heterogeneity at the trial methodology level could be due to lack of standardized chest tube management algorithms required in most trial protocols. Additional reasons at the population level could be patient social factors influencing discharge, such as baseline mobility, physical conditioning, and social support. Not all studies reported patient comorbidities, however the selection bias from for any difference in baseline comorbid disease is

Table 2 RCTs LOS and secondary outcomes random effects meta-analysis results

\begin{tabular}{lcccc}
\hline Outcome & No. trials & Pooled mean difference $(95 \% \mathrm{Cl})$ & Heterogeneity $\left(\mathrm{I}^{2} \%\right)$ & $\mathrm{P}$ \\
\hline LOS & 9 & $-0.960(-1.737,-0.182)$ & $97.4 \%$ & 0.016 \\
Duration air leak & 9 & $-1.152(-1.774,-0.529)$ & $95.3 \%$ & $<0.001$ \\
Duration of chest drainage tube & 7 & $-1.093(-1.612,-0.573)$ & $89.9 \%$ & $<0.001$
\end{tabular}

RCTs, randomized controlled trials; LOS, length of hospital stay; $95 \% \mathrm{Cl}, 95 \%$ confidence interval.

Table 3 RCTs PAL and adverse events random effects meta-analysis results

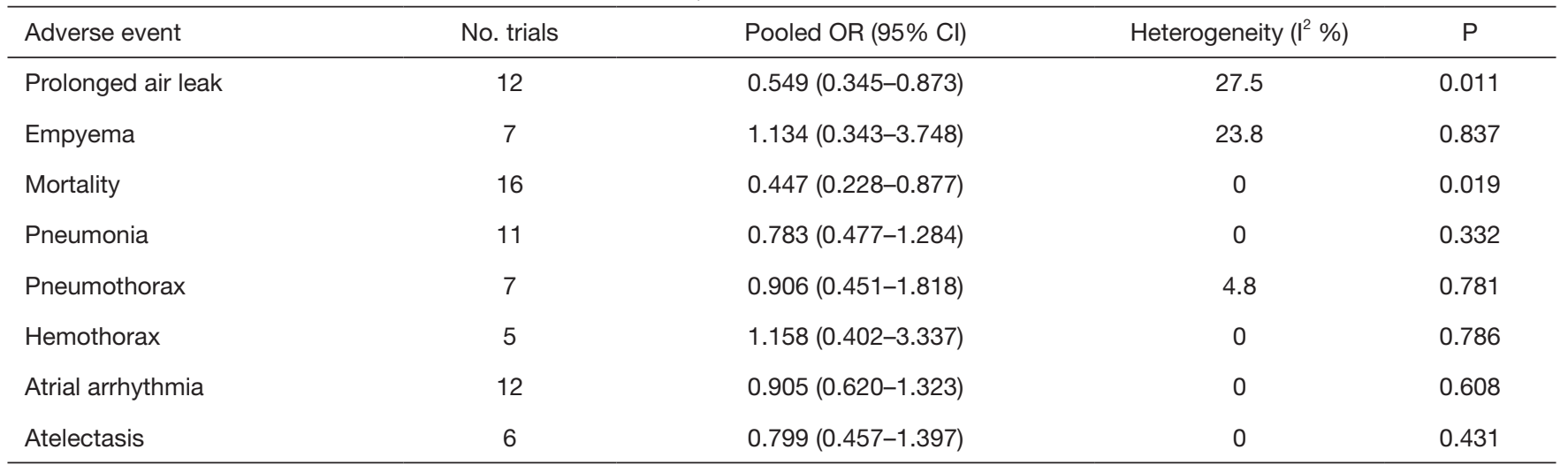

No, number; OR, odds ratio; RCTs, randomized controlled trials; PAL, prolonged alveolar air leak; 95\% Cl, 95\% confidence interval. 
presumably controlled for with randomization. Different procedures included in the pooled analysis (wedge resection, segmentectomy, lobectomy, bilobectomy), although representative of thoracic surgical clinical practice, may also be a source of inter-trial heterogeneity.

Reasons for the lack of statistical significance in many individual RCTs on LOS in contrast to this pooled analysis are likely multi-factorial. Because most air leaks after anatomic pulmonary resection are clinically innocuous and not prolonged, they are managed conservatively with routine chest tube drainage. Study site differences in postoperative patient care and chest drain management may also have contributed, as these algorithms not included in many protocols. For instance, several trials suggest placing chest tubes on water seal may resolve air leaks more rapidly than suction, and this may in turn affect when a chest tube is removed and patient discharge from hospital $(6,11)$.

In addition to lack of precision in postoperative chest tube management, the lack of effect on LOS may be simply due to insufficient statistical power to detect a meaning difference in a secondary outcome measure for individuallevel RCT data.

With respect to post-operative adverse events related to the surgical sealant, we found no evidence of increased odds of empyema on pooled analysis. This is in contrast to the sole RCT reporting 4 cases of pleural sepsis from surgical sealant requiring additional drainage measures (30). The study involved use of a hydrogel-based synthetic surgical lung sealant. Five other RCTs individually did not show similar increases in pleural sepsis, consistent with our weighted findings (31-35). Additionally, there was no evidence of increased odds of any serious adverse event with use of polymeric sealant (mortality, pneumonia, pneumothorax, hemothorax, arrhythmia, or atelectasis) on pooled analysis.

It should be noted that in the vast majority of these included trials, the presence of intra-operative air leak was a requirement for study inclusion. This assessment is important, as it is representative of those patients in clinical practice most likely to benefit from sealant use.

We do not have sufficient evidence to support routine use in all lung resection patients, however the results appear to support a beneficial role for use of sealants intra-operatively in those demonstrating an intra-operative air leak despite standard control measures, or those at known higher than average risk of PAL (presence of emphysema, dense pleural adhesions, LVRS, or incomplete fissure) $(24,37,38)$.

The role of polymeric sealant is unclear in the setting of VATS, as vast majority of trials were conducted with open thoracotomy incisions. In just 2 trials was the VATS approach employed. The first trial examined polymeric sealant in LVRS patients, a known high risk for PAL population (37). The second trial was excluded from metaanalysis because the control group involved use of a fibrin sealant (not standard care alone) (40). Given that the LVRS trial cases were conducted VATS with a significant reduction in rate of PAL demonstrated, this suggests the results may be generalizable to higher risk VATS lobectomy patients. Because VATS is the modern practice standard for pulmonary resections, especially in emphysema patients with lung cancer at risk of PAL, further study is warranted still to determine whether there is a role for routine VATS lobectomy.

\section{Conclusions}

Pooled analysis revealed on average a one day decrease in LOS, duration of air leak and duration of chest tubes with addition of polymeric surgical sealant to standard care. It also revealed decreased odds of PAL with use of polymerizing sealants, without evidence of increased odds of adverse events including empyema. There is evidence of publication bias in the pooled analyses, however sealants may be most beneficial in the high-risk patient with poor quality of lung parenchyma or incomplete lobar fissures, and patients requiring LVRS, or re-operative pulmonary surgery.

\section{Acknowledgements}

The authors extend gratitude to Mr. Glenn Pabilona for administrative support of this paper.

\section{Footnote}

Conflicts of Interest: The authors have no conflicts of interest to declare.

Ethical Statement: This study is a literature review, so ethical review and informed consent of patients are not required.

\section{References}

1. Drewbrook C, Das S, McGuire A, et al. Incidence Risk and Independent Predictors of Prolonged Air Leak in 269 Consecutive Pulmonary Resection Patients over Nine 
Months: A Single-Center Retrospective Cohort Study. Open J Thorac Surg 2016;6:33-46.

2. Belda-Sanchís J, Serra-Mitjans M, Iglesias Sentis M, et al. Surgical sealant for preventing air leaks after pulmonary resections in patients with lung cancer. Cochrane Database Syst Rev 2010;(1):CD003051.

3. Okereke I, Murthy SC, Rice TW, et al. Characterization and importance of air leak after lobectomy. Ann Thorac Surg 2005;79:1167-73.

4. Varela G, Jimenez MF, Aranda JL, et al. Estimating hospital costs attributable to prolonged air leak in pulmonary lobectomy. Eur J Cardiothorac Surg 2005;27:329-33.

5. Brunelli A, Xiume F, Sabbatini A, et al. Air leaks after lobectomy increase the risk of empyema but not of cardiopulmonary complications: a case-matched analysis. Chest 2006;130:1150-6.

6. Cerfolio RJ, Bass CS, Katholi CR, et al. Predictors and treatment of persistent air leaks. Ann Thorac Surg 2002;73:1727-30.

7. McKenna RJ Jr, Fischel RJ, Brenner M, et al. Use of the Heimlich valve to shorten hospital stay after lung reduction surgery for emphysema. Ann Thorac Surg 1996;61:1115-7.

8. DeCamp MM, Blackstone EH, Meli YM, et al. Patient and surgical factors influencing air leak after lung volume reduction surgery: lessons learned from the National Emphysema Treatment Trial. Ann Thorac Surg 2006;82:197-206; discussion 206-7.

9. Fishman A, Martinez F, Naunheim K, et al, National Emphysema Treatment Trial Research Group. A randomized trial comparing lung-volume-reduction surgery with medical therapy for severe emphysema. $\mathrm{N}$ Engl J Med 2003;348:2059-73.

10. Naunheim KS, Wood DE, Mohsenifar Z, et al. Long-term follow-up of patients receiving lung-volume-reduction surgery versus medical therapy for severe emphysema by the National Emphysema Treatment Trial Research Group. Ann Thorac Surg 2006;82:431-43.

11. Brunelli A, Monteverde M, Fianchini A, et al. Predictors of prolonged air leak after pulmonary lobectomy. Ann Thorac Surg 2004;77:1205-10.

12. Isowa $\mathrm{N}$, Hasegawa $\mathrm{S}$, Wada $\mathrm{H}$, et al. Preoperative risk factors for prolonged air leak following lobectomy or segmentectomy for primary lung cancer. Eur J Cardiothorac Surg 2002;21:951-4.

13. Stolz AJ, Schutzner J, Pafko P, et al. Predictors of prolonged air leak following pulmonary lobectomy. Eur J
Cardiothorac Surg 2005;27:334-6.

14. Abolhoda A, Liu D, Burt M, et al. Prolonged air leak following radical upper lobectomy: an analysis of incidence and possible risk factors. Chest 1998;113:1507-10.

15. Andreetti C, Venuta F, Anile M, et al. Pleurodesis with an autologous blood patch to prevent persistent air leaks after lobectomy. J Thorac Cardiovasc Surg 2007;133:759-62.

16. Ng T, Ryder BA, Cioffi WG, et al. Decreasing the incidence of prolonged air leak after right upper lobectomy with the anterior fissureless technique. J Thorac Cardiovasc Surg 2010;139:1007-11.

17. Carboni GL, Vogt A, Dutly AE, et al. Reduction of airspace after lung resection through controlled paralysis of the diaphragm. Eur J Cardiothorac Surg 2008;33:272-5.

18. De Giacomo T, Rendina EA, Coloni GF, et al. Pneumoperitoneum for the management of pleural air space problems associated with major pulmonary resections. Ann Thorac Surg 2001;72:1716-9.

19. Temes RT, Willms CD, Wernly JA, et al. Fissureless lobectomy. Ann Thorac Surg 1998;65:282-4.

20. Hazelrigg SR, Boley TM, Naunheim KS, et al. Effect of bovine pericardial strips on air leak after stapled pulmonary resections. Ann Thorac Surg 1997;63:1573-5.

21. Stammberger U, Klepetko W, Stamatis G, et al. Buttressing the staple line in lung volume reduction surgery: a randomized three-center study. Ann Thorac Surg 2000;70:1820-5.

22. Malapert, Guislain et al. Surgical Sealant for the Prevention of Prolonged Air Leak After Lung Resection: Meta-Analysis. Ann Thorac Surg 2010;90:1779-85.

23. Belboul A, Dernevik L, Roberts D, et al. The effect of autologous fibrin sealant (Vivostat ${ }^{\circledR}$ ) on morbidity after pulmonary lobectomy: a prospective randomised, blinded study. Eur J Cardiothorac Surg 2004;26:1187-91.

24. Rena O, Papalia E, Mineo TC, et al. Air-leak management after upper lobectomy in patients with fused fissure and chronic obstructive pulmonary disease: a pilot trial comparing sealant and standard treatment. Interact Cardiovasc Thorac Surg 2009;9:973-7.

25. Moher D, Liberati A, Tetzlaff J, et al. Preferred reporting items for systematic reviews and meta-analyses: the PRISMA statement. Int J Surg 2010;8:336-41.

26. Higgins JPT, Green S, editors. Cochrane handbook for systematic reviews of interventions, 2011. The Cochrane Collaboration. Available online: http://www.cochranehandbook.org

27. Singhal S, Ferraris VA, Shrager JB, et al. Management of alveolar air leaks after pulmonary resection Ann Thorac 
Surg 2010;89:1327-35.

28. Higgins JP, Thompson SG, Altman DG, et al. Measuring inconsistency in meta-analyses. BMJ 2003;327:557-60.

29. Egger M, Davey Smith G, Schneider M, et al. Bias in meta-analysis detected by a simple graphical test. BMJ 1997;315:629-34.

30. Porte HL, Jany T, Akkad R, et al. Randomized controlled trial of a synthetic sealant for preventing alveolar air leaks after lobectomy. Ann Thorac Surg 2001;71:1618-22.

31. De Leyn P, Muller MR, Sokolow Y, et al. Prospective European multicenter randomized trial of PleuraSeal for control of air leaks after elective pulmonary resection. J Thorac Cardiovasc Surg 2011;141:881-7.

32. Wain JC, Kaiser LR, Johnstone DW, et al. Trial of a novel synthetic sealant in preventing air leaks after lung resection. Ann Thorac Surg 2001;71:1623-8.

33. Lequaglie C, Marasco RD, Giudice G. Use of a sealant for prevention of prolonged air leaks recorded by a digital chest drain system after lung resection. A prospective randomized study. J Cardiothorac Surg 2012;7:106.

34. D'Andrilli A, Andreetti C, Ibrahim M, et al. A prospective randomized study to assess the efficacy of a surgical sealant to treat air leaks in lung surgery. Eur J Cardiothorac Surg 2009;35:817-20.

35. Tan C, Utley M, Paschalides C, et al. A prospective randomized controlled study to assess the effectiveness of CoSealW to seal air leaks in lung surgery. Eur J Cardiothorac Surg 2011;40:304-8.

36. Wong K, Goldstraw P. Effect of fibrin glue in the reduction of postthoracotomy alveolar air leak. Ann Thorac Surg 1997;64:979-81.

37. Moser C, Opitz I, Zhai W, et al. Autologous fibrin sealant reduces the incidence of prolonged air leak and duration of chest tube drainage after lung volume reduction surgery: a prospective randomized blinded study. J Thorac Cardiovasc Surg 2008;136:843-9.

38. Marta GM, Facciolo F, Ladegaard L, et al. Efficacy and safety of TachoSilW versus standard treatment of air leakage after pulmonary lobectomy. Eur J Cardiothorac Surg 2010;38:683-9.

39. Lang G, Csekeo A, Stamatis G, et al. Efficacy and safety of topical application of human fibrinogen/ thrombincoated collagen patch (TachoComb) for treatment of air leakage after standard lobectomy. Eur J Cardiothorac Surg 2004;25:160-6.

40. Kılıç B, Erşen E, Demirkaya A, et al. A prospective randomized trial comparing homologous and autologous fibrin sealants for the control of alveolar air leak. J Thorac Dis 2017;9:2915-22.

41. Gonfiotti A, Santini PF, Cardillo G, et al. Safety and Effectiveness of a New Fibrin Pleural Air Leak Sealant: A Multicenter, Controlled, Prospective, ParallelGroup, Randomized Clinical Trial. Ann Thorac Surg 2011;92:1217-24; discussion 1224-5.

42. Filosso PL, Ruffini E, Sandri A, et al. Efficacy and safety of human fibrinogen-thrombin patch (TachoSil@) in the treatment of postoperative air leakage in patients submitted to redo surgery for lung malignancies: a randomized trial. Interact Cardiovasc Thorac Surg 2013;16:661-6.

43. Fabian T, Federico JA, Ponn RB. Fibrin glue in pulmonary resection: a prospective, randomized, blinded study. Ann Thorac Surg 2003;75:1587-92.

44. Cardillo G, Carleo F, Gonfiotti A, et al. Adverse effects of fibrin sealants in thoracic surgery: the safety of a new fibrin sealant: multicentre, randomized, controlled, clinical trial+. Eur J Cardiothorac Surg 2012;41:657-62.

45. Belcher E, Dusmet M, Jordan S, et al. A prospective, randomized trial comparing BioGlue and Vivostat for the control of alveolar air leak. J Thorac Cardiovasc Surg 2010;140:32-8.

46. Anegg U, Lindenmann J, Matzi V, et al. Efficiency of fleece-bound sealing (TachoSil) of air leaks in lung surgery: a prospective randomised trial. Eur J Cardiothorac Surg 2007;31:198-202.

47. Allen MS, Wood DE, Hawkinson RW, et al. Prospective randomized study evaluating a biodegradable polymeric sealant for sealing intraoperative air leaks that occur during pulmonary resection. Ann Thorac Surg 2004;77:1792-801.

48. Tansley P, Al-Mulhim F, Lim E, et al. A prospective, randomized, controlled trial of the effectiveness of BioGlue in treating alveolar air leaks. J Thorac Cardiovasc Surg 2006;132:105-12.

49. McGuire AL, Petrcich W, Gilbert S, et al. Digital versus analogue pleural drainage phase 1: prospective evaluation of interobserver reliability in the assessment of pulmonary air leaks. Interact Cardiovasc Thorac Surg 2015;21:403-7.

Cite this article as: McGuire AL, Yee J. Clinical outcomes of polymeric sealant use in pulmonary resection: a systematic review and meta-analysis of randomized controlled trials. J Thorac Dis 2018;10(Suppl 32):S3728-S3739. doi: 10.21037/ jtd.2018.10.48 